\title{
Article \\ Electrical Breakdown Spectroscopy of Nano-/Micro-Thermites
}

\author{
Stefan Lundgaard ${ }^{1, *,+} \oplus$, Soon Hock $\mathrm{Ng}^{1, *,+}{ }^{\mathbb{D}}$, Damien Cahill ${ }^{2}$, Johan Dahlberg ${ }^{2}$, Jamie Allender ${ }^{2}$, \\ Michael Barber ${ }^{2}$, Joshua Stephens ${ }^{2}$ and Saulius Juodkazis ${ }^{1,3}$ (D) \\ 1 Optical Sciences Centre and ARC Training Centre in Surface Engineering for Advanced Materials (SEAM), \\ School of Science, Swinburne University of Technology, Hawthorn, VIC 3122, Australia; \\ sjuodkazis@swin.edu.au \\ 2 DefendTex Pty Ltd., 46 Bessemer Drive, Dandenong South, VIC 3175, Australia; \\ damien.cahill@defendtex.com (D.C.); johan.dahlberg@defendtex.com (J.D.); \\ jamie.allender@defendtex.com (J.A.); michael.barber@defendtex.com (M.B.); \\ joshua.stephens@defendtex.com (J.S.) \\ 3 Tokyo Tech World Research Hub Initiative (WRHI), School of Materials and Chemical Technology, \\ Tokyo Institute of Technology, 2-12-1, Ookayama, Meguro-ku, Tokyo 152-8550, Japan \\ * Correspondence: slundgaard@swin.edu.au (S.L.); soonhockng@swin.edu.au (S.H.N.) \\ + These authors contributed equally to this work.
}

check for updates

Citation: Lundgaard, S.; Ng, S.H.; Cahill, D.; Dahlberg, J.; Allender, J.; Barber, M.; Stephens, J.; Juodkazis, S. Electrical Breakdown Spectroscopy of Nano-/Micro-Thermites. Technologies 2021, 9, 34. https://doi.org/10.3390/ technologies 9020034

Academic Editor: Manoj Gupta

Received: 12 April 2021

Accepted: 21 April 2021

Published: 29 April 2021

Publisher's Note: MDPI stays neutral with regard to jurisdictional claims in published maps and institutional affiliations.

Copyright: (c) 2021 by the authors. Licensee MDPI, Basel, Switzerland. This article is an open access article distributed under the terms and conditions of the Creative Commons Attribution (CC BY) license (https:// creativecommons.org/licenses/by/ $4.0 /)$.
Abstract: We describe the optical and electrical analysis of a micro-explosion of silicon and microthermite micro-bridges with a spectral temporal resolution within a $2 \mathrm{~ms}$ time range. The intensity of peaks and their mean lifetimes are calculated to identify the contributing atomic and molecular species. Singly ionised atoms and molecules were the main contributor to spectroscopic signature of emission. It is shown that micro-bridges can be used to characterise small (safe) $<10 \mathrm{mg}$ amounts of energetic materials. Use of ions at low ionisation numbers of $\mathrm{Si}$ as well as Cu-oxides with different metal-to-oxygen ratios allows the thermal conditions for micro-explosions and a higher temperature of the discharge plasma to be engineered. Temperatures of $5000 \pm 500 \mathrm{~K}$ (the black-body radiation fit) were achieved with simple micro-bridge junctions of $10-30 \Omega$ resistance initiated with 100-250 V (triggered capacitor discharge). The demonstrated approach can be applied in material science research concerning ultra-fast melting, phase transitions, and detailed steps leading towards detonation (exponentially growing exothermic chemical reactions).

Keywords: breakdown spectroscopy; nano-/micro-thermites

\section{Introduction}

Plasma research is active in all fundamentally important directions, such as future energy generation with inertial confinement fusion (ICF) [1], as a non-linear medium for increasing laser intensity levels beyond chirped pulse amplification (CPA) [2], the exploration of light-matter interactions at relativistic conditions with high power, terawatt (TW) laser pulses [3], extreme ultra-violet (EUV) nanolithography light sources at a wavelength of $13.5 \mathrm{~nm}$, which use laser-induced breakdown (LIB) of liquid Sn droplets [4], wakefield plasma accelerators [5,6], and plasma propulsion of spacecraft [7]. Plasma, the 4 th state of matter, is the most energetic of the four states. Its creation requires enough energy to break chemical bonds and ionise matter. Concentrated energy delivery is usually achieved via focused laser pulses or electrical breakdown by large electrical fields in engineered circuitry. In the case of ultra-short sub-1 ps laser pulses, material transition from dielectric to metallic can be tailored by calculated absorbed energy delivery; hence, a die-met [8] state of matter can be created as defined by the permittivity $\varepsilon$.

The largest amount of energy delivery (release) in the shortest time is sought after for laser initiation. The explosion can be characterised by its acoustics (pressure) and light emission signatures [9]. Time-resolved spectroscopy is the usual method of choice for remote, non-contact detection of spectral lines of ions and excited molecules/atoms. Laser-induced breakdown spectroscopy (LIBS) is a reliable technique to detect elements with $1 \%$ wt. sensitivity in a remote $(1.5 \mathrm{~m})$ manner. The first LIBS instrument in space was 
famously the ChemCam instrument on the Mars Science Laboratory (Curiosity rover) and others have been proposed for lunar missions [10,11]. Using short, picosecond pulses and higher irradiation intensities, LIBS was used in combination with shadowgraphy to reveal fast ablation/explosion dynamics [12].

Here, time-resolved spectral analysis of the optical emission from plasmas made by large discharge currents in silicon and nano-thermite laminate micro $(\mu)$-bridge structures are investigated $[13,14]$. Voltage, current transients, together with optical emissions were monitored as the plasma volumes evolved from tens-of-micrometers in cross-section to the $\mathrm{mm}$ scale. Comparisons of the energy efficiency of micro-bridges triggered electrically and by optical initiation of plasma can be made and are expected to converge for small sizes. A small micro-bridge footprint is favourable to compare energy delivery to Joule heating by current and by light. Investigation of new materials for electrical and optical initiation of explosions is the current trend due to harmful effects of lead-based chemicals and the challenges of safe storage [15].

\section{Experimental}

Micro-bridges shaped as bowties offer a controlled method for electrical decomposition by controlling the dimensions of a bridge connection between two contact pads [13]. The designs were made by standard contact photolithography of AZ1518 resist at $1.8 \mu \mathrm{m}$ thickness and used as a mask for etching for silicon or for sputtered and e-beam evaporated layered nano-thermites [15]. The masks were made on an intelligent micro-patterning (IMP) SF100 XPRESS, which uses a micro-mirror array $(1024 \times 768)$ and a UV lamp for exposure through a set of optics. This method allows for improved overlay of highresolution micro-bridge regions connected with large area contact pads using different projection magnifications.

The fabricated bridges were glued into a printed circuit board (PCB) and wire bonded using a F\&S Bondtec wirebonder with a $25 \mu \mathrm{m}$ diameter 99\% Al-1\% Si alloy wire from Heraeus Group with a DeWeyl Tool Company CCNVE-1/16-1"-45-C-2020-M bond wedge. The Al wires were attached to the contact pads of bridges using the standard process and they were optimised for the best mechanical and electrical contact.

Standardisation of the wire bonds between the bridge contacts and PCB was essential step to establish reliable and repeatable conditions for optical and acoustic characterisation. Figure 1a shows the PCB with electroless nickel immersion gold (ENIG) contacts with mounted and bonded bridges in between, and Figure $1 \mathrm{~b}$ shows a developed micro-bridge pattern before etching or layered deposition of different thermite materials $\mathrm{B}, \mathrm{Al}, \mathrm{Cu}$, or $\mathrm{Cu}$-oxides top. $\mathrm{CuO}$ can be deposited with a controlled $\mathrm{Cu}$-to-O ratio using $\mathrm{O}_{2}$ in the plasma deposition step (b) bottom. For investigation of initiation of energetic chemicals, Si micro-bridges were loaded with boron potassium nitrate $\left(\mathrm{BKNO}_{3}\right)$, a common igniter compound, using drop casting.

The electrical discharge setup was conceptually the same as described in a previous study [16]. A capacitor at a set voltage was discharged across the $\mu$-bridge, causing Joule heating of the silicon or thermite. Simultaneously, current and voltage transients were recorded using a differential probe connected parallel to the bridge and a current clamp meter over the positive lead. In some experiments, high-speed video, integrated spectra, high-speed spectra, and acoustic pressure signature were also recorded.

Spectral characterisation was carried out with a portable setup based on a Ocean Optics (Ocean-FX-VIS-NIR-ES, spectral resolution $\sim 2.5 \mathrm{~nm}$ ) portable spectrometer and laptop computer. Each acquisition consists of the integration time plus a fixed readout and processing time of $211.25 \mu \mathrm{s}$. Hence, with a minimum integration time of $10 \mu \mathrm{s}$, the spectrometer achieves a maximum of $\sim 4500$ scans per second. The plasma emission during the rising stage and the decaying part are complex but can usually be fitted by an exponential dependence of the number density of emitters, the excited ions/atoms: $N(t)=N_{0} \exp (-\lambda t)$, where $N_{0}$ is the initial density at $t=0$ and $\lambda$ is the exponential decay constant; $\tau=1 / \lambda$ is the mean lifetime for of emitters decaying to the $1 / e \approx 36.8 \%$ of initial level. 
(a)

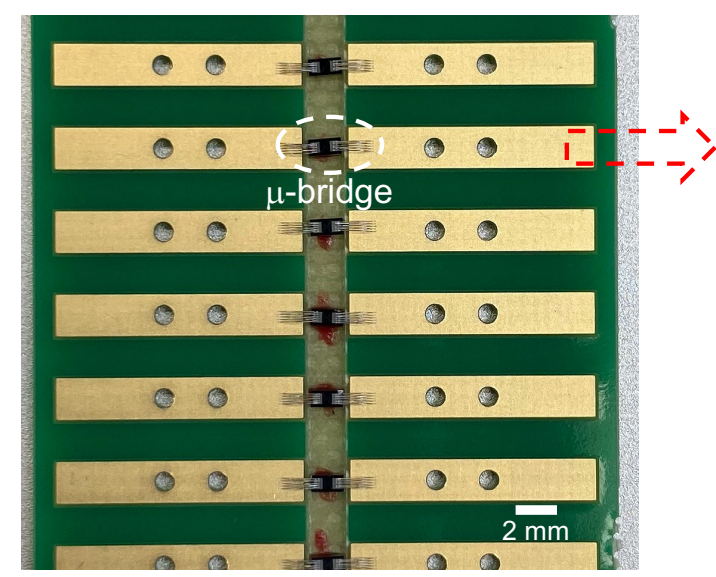

(b)

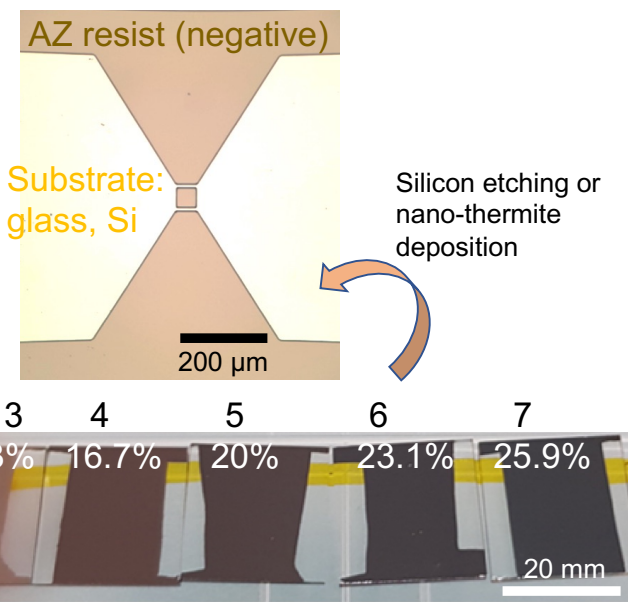

Figure 1. (a) $\mu$-bridge (lithographically defined silicon or nano-thermite layers) glued and wire-bonded in a PCB mount with accessible contact pads at either end. A capacitor is discharged across the contacts with the voltage measured in parallel using a differential probe and current measured with a clamp meter. (b) Top: optical micrograph of one of the designs of the $\mu$-bridge defined in the AZ1505 resist after exposure and development; the typical width of the bridge connection was $\sim 50 \mu \mathrm{m}$. Etching of the silicon was performed using such resist patterns on the substrate (glass or $\mathrm{Si}$ ) as was the deposition of nano-thermites. Bottom: $\mathrm{CuO}$ films deposited with varying proportions of $\mathrm{O}_{2}$ in the Ar carrier gas during plasma sputtering.

\section{Results and Discussion}

\subsection{Optical Signature of Electrical $\mu$-Thermite's Breakdown}

The Joule heating in a resistor of resistance, $R[\Omega]$, for an ideal case when the electrical current, $I[\mathrm{~A}]$, is all consumed through heating is defined by the power $P=U \cdot I=$ $I \cdot R^{2}=U / R^{2}$, where $U[\mathrm{~V}]$ is the applied voltage. By applying direct current (DC) of capacitor discharge [16], bridges of $R=4-1000 \Omega$ made of different material layers were initiated. A typical time-resolved emission of an electrically triggered bridge is shown in a map (Figure 2a) with the highest intensity spectrum $(t=623 \mu \mathrm{s})$ shown in Figure $2 \mathrm{~b}$. The temporal resolution was $312 \mu$ s with an integration time of $100 \mu \mathrm{s}$. The experimental spectrum is overlaid with theoretical LIBS spectrum for the chemical elements used on the bridges [17]. The NIST spectra at typical conditions, i.e., a vacuum, $1 \mathrm{eV}$ temperature, and $10^{17} \mathrm{~cm}^{-3}$ density, were used. It is known that atmospheric conditions can cause stronger background emission in LIBS [18]. Some of the strongest $\mathrm{Cu}$ I atomic emission lines match the spectral lines in the $510-530 \mathrm{~nm}$ spectral window. Theoretical LIBS spectra for $\mathrm{K} \mathrm{I}$ emission was to found match the strongest doublet line at 767 and $770 \mathrm{~nm}$ within $1 \mathrm{~nm}$ (Figure 3a). Furthermore, the intensity ratio between the peaks was qualitatively reproduced; see the inset in Figure 3a.

The time evolution of the optical emission has rising and decaying sections, which can be visually enhanced using $\lg (t)$ scale (Figure 2a). Apparently, the strongest $\mathrm{K}$ I and Al I lines (from chemical explosive, thermite, and wire bond components) can be seen from the very first recorded spectra. Figure $3 \mathrm{~b}$ shows a single exponential fit to the decay side of the emission. It had a time constant of $0.125 \mathrm{~ms}$. Spectral shift and broadening due to thermal velocity of the emitting atoms and ions was not expected to change the spectra. Indeed, the Doppler shift is $\omega=\omega_{0} \times\left(1 \pm v_{T} / c\right)$, where $\omega_{0}=2 \pi c / \lambda_{0}$ is the cyclic frequency at rest, $c$ is speed of light, $v_{T}$ is the thermal velocity of emitter, and $\lambda_{0}$ is the wavelength for atom/ion emitting at rest; the sign " + " corresponds to the source moving towards the detector (blue-shifted), while " - " is the source departing from detector (red-shifted). The thermal velocity of emitters causes spectral broadening $\frac{\Delta \lambda}{\lambda}=2 \sqrt{2 \ln 2 \frac{k_{B} T}{m_{0} c^{2}}}$, where $k_{B}$ is the Boltzmann constant, and $m_{0}$ is the rest mass of the emitter at the temperature (absolute) $T[\mathrm{~K}]$. 

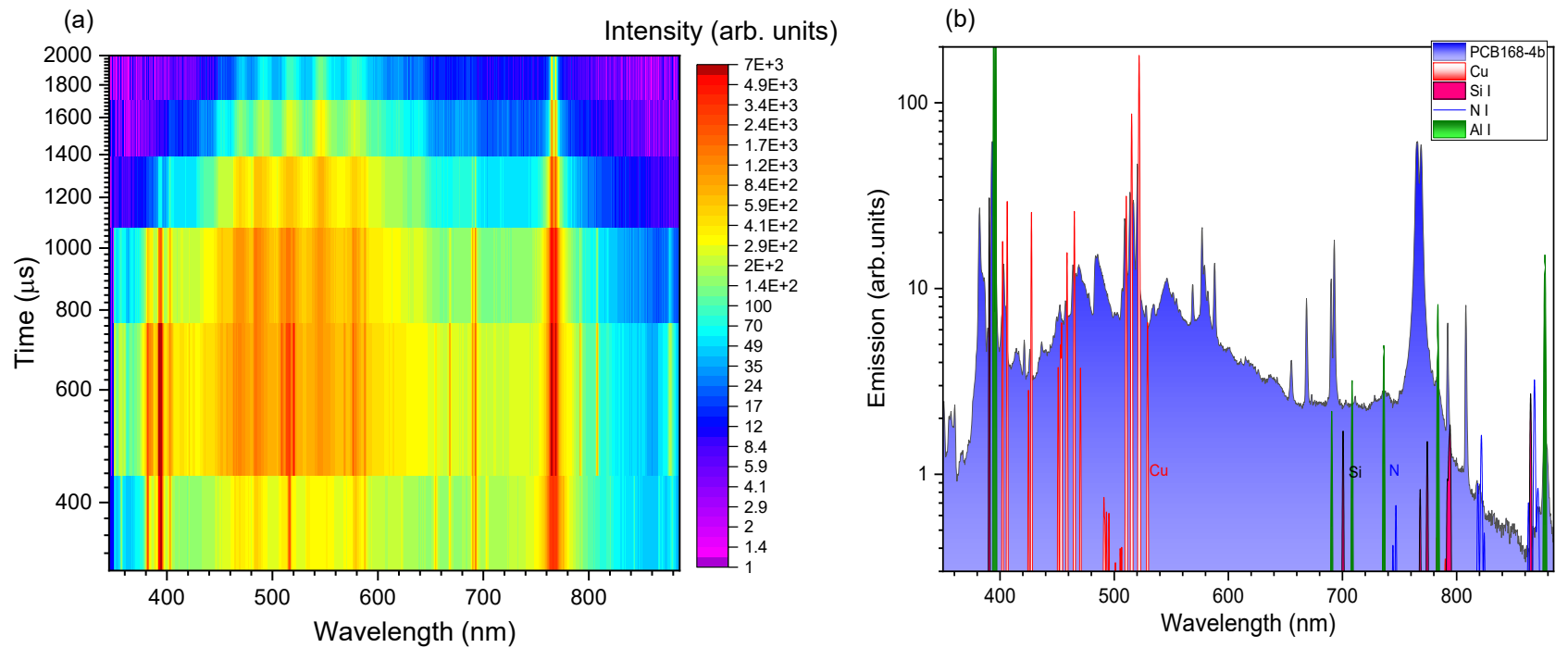

Figure 2. (a) Temporal evolution of emission inducedby electrical breakdown of a silicon bridge with $\mathrm{BKNO}_{3}$ on the $\mathrm{PCB}$ substrate. The integration time was $100 \mu$ s with a sampling interval of $312 \mu$ s. The intensity and time are presented in log-scale. (b) Spectra at time slice $t=623 \mu$ s overlaid with atomic emission lines of Cu I, Si I, N I Al I (data from NIST database for light breakdown spectroscopy LIBS; I indicates atomic spectra, and II indicates single ionisation spectra). Each intensity plot has different counts and is presented for better visualisation of the overlap of peaks.
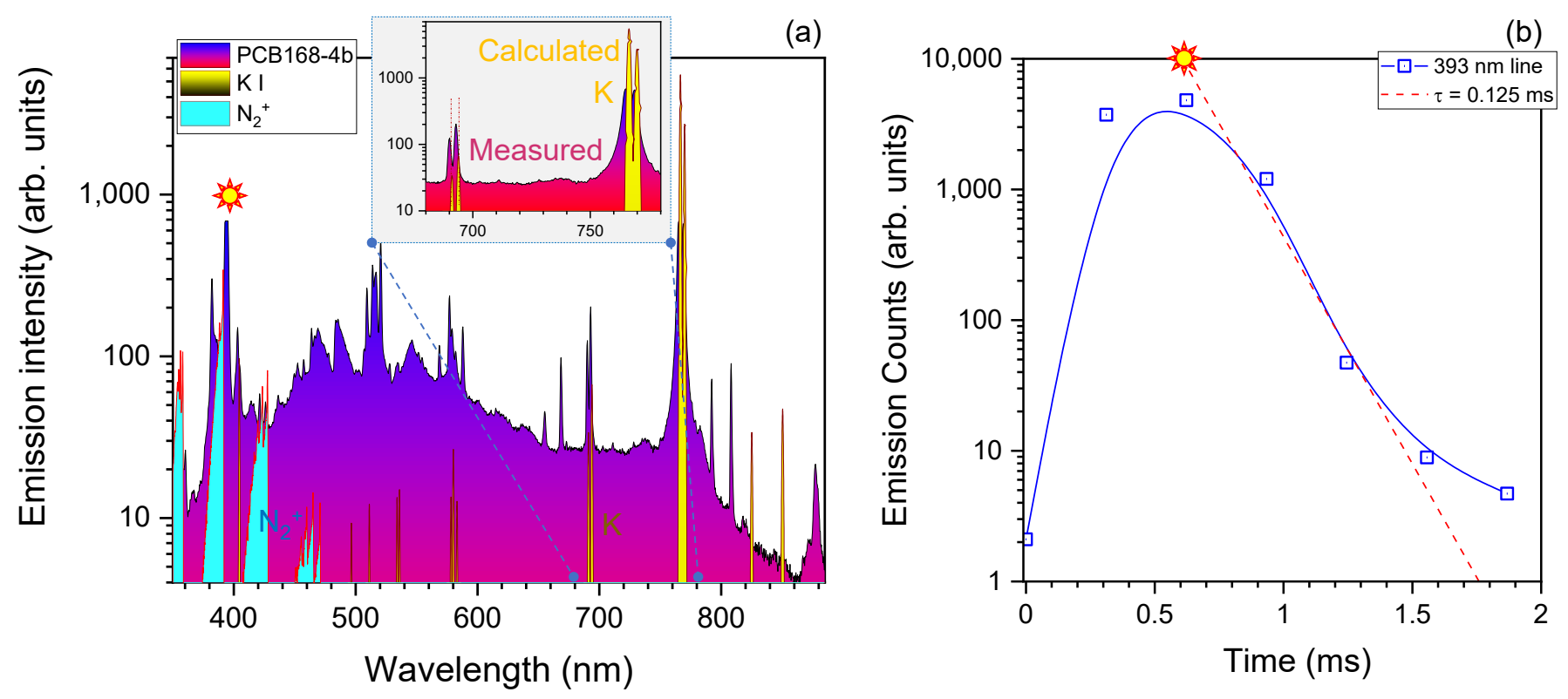

Figure 3. (a) Emission of thermitePCB 168-4b (same as Figure 1b) overlayed with K I emission from LIBS (NIST database [17]). The inset shows match between the predicted spectral positions of the K I (I for the neutral element) lines and experimentally measured thermite emission. For illustration of molecular spectral shape, the emission of the molecular $\mathrm{N}_{2}^{+}$ion at $4000 \mathrm{~K}$ is shown (Specair 3.0). (b) Temporal evolution of the $393 \mathrm{~nm}$ band (matching peak emission of Al I from the $\mu$-thermite and $\mathrm{N}_{2}^{+}$from the loaded chemical $\mathrm{BKNO}_{3}$ ). The one-exponential fit with $\tau=0.125 \mathrm{~ms}$ (dashed line).

Electrical discharge and breakdown of a micro-bridge with a thermite-layered structure and silicon with explosive chemicals on top progresses over several stages. After heating, melting, and evaporation driven by electrical current, the further steps progress via an ionisation and electrical discharge arc through the expanding electrically conductive plasma, which is fed by consumption of the thermite or $\mathrm{BKNO}_{3}$. These complex dynamics 
depend on properties of electrodes, their surface, the amount of fuel, and the ambient environment. Surface charge movement and air ionisation can be modelled with high accuracy [19]. Once the gap between electrodes is opened, the density of ion species is defined by the power $P_{a b s}$ absorbed by the discharge gap (the formula for a plasma thruster) [20]:

$$
n_{\text {ions }}=\frac{P_{a b s}}{e u_{B} S_{0} \epsilon_{T}}=\frac{P_{a b s}}{e^{3 / 2} \sqrt{T_{e} / M} S_{0} \epsilon_{T}},
$$

where $u_{B}=\sqrt{e T_{e} / M}$ is the Bohm velocity, $M$ is the ion mass, $T_{e}$ is the electron temperature, $e$ is its charge, $S_{0}$ is the effective area from which an ion-electron pair is lost, $\epsilon_{T}$ is the total energy lost per electron-ion pair leaving a plasma arc. This shows the basic scaling that if high-energy electrons are leaving the cross-sectional region of the arc discharge, there is lesser absorbed energy and reduced production of ions.

The temperature of the plasma can be estimated from the black body radiation fit of a broad spectrum (the linearised form Emission $\times \lambda^{2} \propto \hbar \omega[\mathrm{eV}]$ [21]. See Appendix A for more detail.) and, more precisely, from a fit of the rotational manifold of molecular spectra, e.g., $\mathrm{N}_{2}, \mathrm{~N}_{2}^{+}$in flame plasma [22] or electrical discharge plasma [23] (see the $\mathrm{N}_{2}^{+}$spectral signature in Figure $3 \mathrm{a}$ ). The rate of chemical reactions, convection, and charge injection (electrical discharge) can generate plasmas with species out of thermal equilibrium with different temperatures characterising vibrational and rotational degrees of freedom [22].

\subsection{Electrical Signature of Plasma Formation}

Figure $4 \mathrm{a}, \mathrm{b}$ compares the time evolution of a $220 \mu \mathrm{F} U=100 \mathrm{~V}$ capacitor discharge through a micro-bridge in free space and loaded with $<10 \mathrm{mg} \mathrm{BKNO}_{3}$. The ignition event was triggered and monitored on an oscilloscope connected to the ENIG contacts (Figure 1a). In both cases, the time span from 0.1-1 $\mu$ s shows the initiation of discharged triggered by a TTL $5 \mathrm{~V}$ signal at a time moment of $\pm 10 \mathrm{~ns}$, representing 0 (Figure 4 ). The oscillations during the first $\sim 2 \mu$ s are artefacts caused by the load-matching and RC-constant of the electronics used. Similar oscillations are seen when a purely restive load was used. In the free space case, Joule heating of the micro-bridge occurs within the first $3 \mu$ s as determined from high-speed video (frame interval $1.54 \mu \mathrm{s}$ ). During this time, the silicon melts then vaporises and subsequently ionises into a plasma as the current heats the vapour. The sharp drop in voltage at $2.3 \mu$ s could be indicative of this transition. As the plasma plume expands, current begins to flow between the wire bonds, causing them to ionise and fuel the plasma. The drop in voltage at $4 \mu$ s and the subsequent increase in the zoomed highspeed video mean pixel intensity at $5 \mu$ s indicates when this occurs. The plume continues to expand until it bridges the electrical contacts, causing the large increase in current as the effective cross-section of the conductive pathway increases, resulting in the capacitor discharging at an increased rate.

For the $\mathrm{BKNO}_{3}$ case (Figure $4 \mathrm{~b}$ ), a fine micro-powder was mixed with a polymer binder in ethanol, drop-cast onto the bridge, then dried in an oven at $50{ }^{\circ} \mathrm{C}$ overnight. This procedure, which is aimed at using minute amounts energetic material on a $\mu$-bridge, was tested in this preliminary study. As before, Joule heating occurs, but there is no appreciable change in voltage in the $2.3-4 \mu$ s range and only a slight increase in current. This suggests that a plasma is not formed at that time due to the additional thermal mass from the $\mathrm{BKNO}_{3}$, which has an ignition point of just under $450{ }^{\circ} \mathrm{C}$ [24]. There is an extended plateau in both voltage and current until $\sim 40 \mu$ s, after which the voltage begins to fluctuate and current begins to increase. During this time, it can be inferred that all the energy is being dissipated into the $\mathrm{BKNO}_{3}$. After $\sim 40 \mu \mathrm{s}$, the high-speed camera pixel intensity begins to increase, coinciding with a jump in current. As this stage, the $\mathrm{BKNO}_{3}$ over the bridge area is ejected and the plasma becomes visible to the camera. Preliminary burn rate tests showed that $\mathrm{BKNO}_{3}$ burned at a rate of $\sim 16 \mathrm{~mm} / \mathrm{s}$ in free space $(0.64 \mu \mathrm{m}$ in $40 \mu \mathrm{s})$, indicating that the electrical discharge is the dominant form of energy sustaining the reaction. The sharp voltage drop and current rise at $250 \mu$ s is indicative of the stage when the ENIG contacts are bridged. The sharper current drop could be due to the lower remaining charge in the capacitor or a drop in conductivity of the ionised material, which decayed single exponentially with the time constant $\tau=0.125 \mathrm{~ms}$ (Figure $3 \mathrm{~b}$ ). This series of events can 
also be seen in the time-series spectra shown in Figure 2, albeit a different bridge and event. In the initial time slice $(t=312 \mu$ s with a $100 \mu$ s integration time and a $312 \mu$ s total processing time), the Al I and $\mathrm{K}$ I lines are clearly visible, showing that the Al wire bonds have been vaporised along with elements of the $\mathrm{BKNO}_{3}$. The $\mathrm{Cu}$ I emission lines between 510-530 nm (Figure 2a) are not evident, suggesting that ENIG pads have yet to be bridged in this case. In the next time slice, $t=648 \mu \mathrm{s}$, the $\mathrm{Cu}$ I lines are very intense, along with even stronger Al I and K I lines. This indicates that the ENIG has been bridged and the pads are vaporising. It is at this stage that there is a maximum emission and the reaction is at its height. As the plume size increases over time, the Al I lines fade much quicker than both the $\mathrm{Cu} \mathrm{I}$ and $\mathrm{K}$ I since the total mass of $\mathrm{Al}$ is much lower than the $\mathrm{Cu}$ in the ENIG pads and the $\mathrm{K}$ in the $\mathrm{BKNO}_{3}$. This typical scenario of (1) thermal breakdown of the bridge during the fast heat-up stage, (2) plasma initiation, (3) ENIG contact ionisation, and (4) decay were repeated for different compositions of $\mu$-bridges with and without explosive loading. The inset in Figure 4 shows $\sim 10 \mathrm{mg} \mathrm{BKNO}_{3}$ burning when contained inside an aluminium holder (see the Supplementary Material for the evaluation of energy release). Optical imaging at different positions of the spark might be required for detailed characterisation of the dynamics and its spectroscopic signatures.
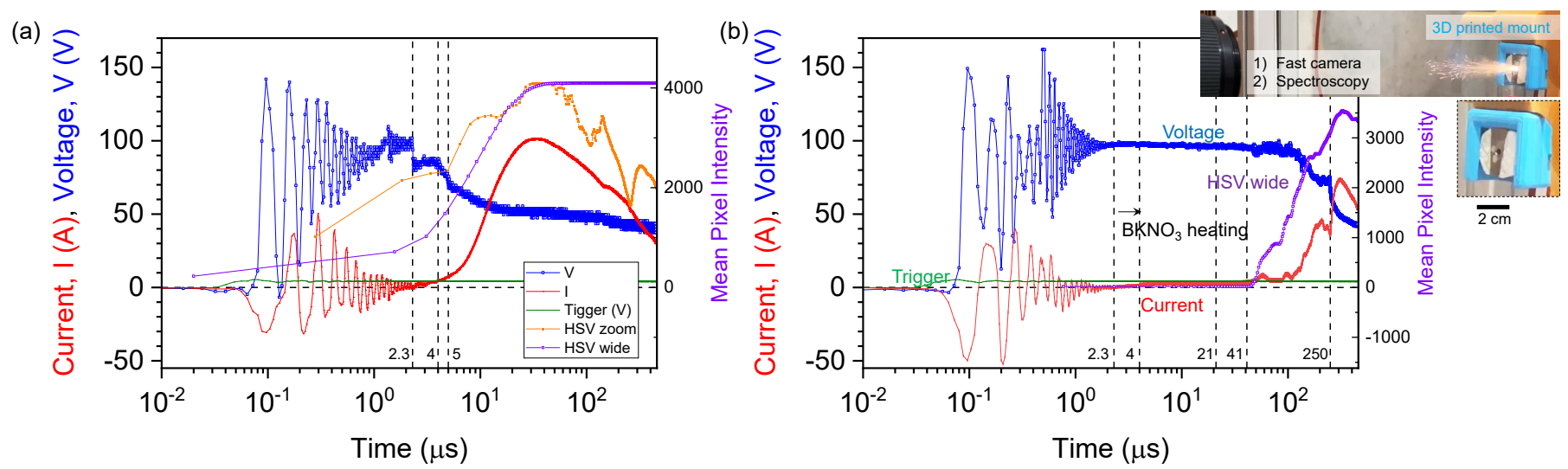

Figure 4. Plots showing the time evolution of current $I$ and voltage $V$ transients for (a) a silicon bridge in free space, $R \approx 18 \Omega$, including mean pixel intensity of high-speed camera video in both a narrow, zoomed field-of-view focused on the bridge and a wide field-of-view focused on an area highlighted with the white dotted circle in Figure 1a (the wide view was from another bridge fired under the same conditions) and (b) a silicon bridge loaded with $<10 \mathrm{mg} \mathrm{BKNO}_{3}$, $R \approx 54 \Omega$, including the mean pixel intensity from a wide field-of-view. The inset shows a phone-captured frame of the explosion event triggered by a $\mu$-bridge loaded with $<10 \mathrm{mg} \mathrm{BKNO}$. The video was taken from another bridge fired under similar conditions. The PCB was mounted in a 3D printed holder for a fixed position to record high-speed video (HSV) and spectroscopic data.

\subsection{Scaling Insights from Analytical Formulae and Applications}

The use of $\mu$-thermite based on layered metal $(\mathrm{Cu}, \mathrm{Al})$, semi-metal $(\mathrm{Zr}, \mathrm{Si})$, and metal oxide, placed on different substrates, e.g., large surface areas such as black-Si [25,26], can tune the oxygen deficiency of energetic materials placed on top. This, in turn, can facilitate faster energy deposition from the thermite to the explosive (See Appendix B for energy balance calculations $[27,28])$. The ohmic conductivity of plasma can be estimated from the electron scattering and ion of atomic number $Z$ (protons), which becomes $\sigma=\frac{3\left(k_{B} T\right)^{3 / 2}}{2 \pi e^{2} Z \sqrt{m / 3}}$, where $k_{B}$ is the Boltzmann constant, $T[\mathrm{~K}]$ is the absolute temperature, and $e$ is the electron charge [29]. This is derived from the Coulomb ion-electron collision cross-section $\sigma_{e i}=$ $\pi r_{0}^{2}=\frac{\pi Z^{2} e^{4}}{m^{2} v^{4}}$ when the $\theta=90^{\circ}$ degree scattering events dominate (as established by experiments), where $v$ is the velocity of the electron $\left(m v^{2} / 2=k T / 2\right), m$ is its mass, and $r_{0}$ is the impact distance. The collision frequency of electrons and ions of density $n_{i}$ is $v_{e i}=n_{i} \sigma_{e i} v=\frac{3^{-3 / 2} \pi e^{4} n_{e} Z}{\sqrt{m}\left(k_{B} T\right)^{3 / 2}}$, with $n_{e}$ being the electron density. Thereafter, the conductivity is defined by the mobility $\mu$ and $\sigma=n_{e} e \mu$, while $\mu=\frac{e}{m} \tau_{e i}=\frac{e}{m} \times \frac{1}{v_{e i}}$; here, $\tau_{e i}$ is the 
time constant of the $\mathrm{e}-\mathrm{i}$ scattering. The more precise formulae accounting for different scattering angles $\theta$ and to the quantum correction when the impact distance $r_{0}$ should be compared with the de Broglie wavelength of electron are all available [29]. However, the formulae presented above show the main scaling rules. Interestingly, the plasma conductivity $\sigma$ is independent of the plasma density $n_{e}$ since $\sigma \propto n_{e} \mu \propto n_{e} / \nu_{e i} \propto n_{e} / n_{e}=1$. The conductivity is strongly dependent on the temperature $\propto T^{3 / 2}$ and is smaller for heavier atoms (larger $Z$ ). However, the plasma can be compared to the highly conductive metals only at temperatures of $T \sim 100 \mathrm{eV}$ encountered in the exotic internal confinement fusion and astronomical plasmas.

The used micro-thermite with a defined area of $\sim 10 \times 10 \mu \mathrm{m}^{2}$ can be easily overlaid on a micro-thermocouple or micro-bolometer deposited by sputtering or evaporation for characterisation of temperature evolution at a specific location on the substrate from the "ground zero" [30]. In addition to the fast heating, the small micro-volume of $\mu$-thermites also provides a fast thermal quenching required to capture non-equilibrium phases of materials as was demonstrated for the retrieval of the amorphous phase of bio-crystalline silk [31]. The interfacial heat conductivity changes around the melting point of $1 \mathrm{ng}$ of indium were measured using a micro-heater [32]. The combined spectral, thermal, and acoustic evolution of fast $\sim 1 \mu$ s events triggered by/on electrically driven $\mu$-thermites, which are engineered with nano-layered energetic metal, semi-metal, and oxides using physical deposition, provides a versatile research toolbox. The most simple projection lithography or direct writing techniques are required for the definition of the $\mu$-thermites.

\section{Conclusions and Outlook}

The time-resolved spectra of electrically initiated thermites made from layers of different materials and loaded with explosive chemicals are presented herein. Working with micro-bridges that are initiated electrically rather mechanically, as is the case with conventional methods, makes it more safe. The layered metallic electrically conductive materials can be made optically non-reflective (black) using a combination of dielectric and metallic layers [33]. This could be envisaged as yet another application of meta-materials for optical primers [15]. It is shown here that micro-thermites with and without loading with explosive chemicals can be safely operated with total load below $1 \mathrm{~g}$. Further miniaturisation in energetic material characterisation can be envisaged due to the availability of microspectrometers [34]. Space-time imaging techniques based on computational holography using pinhole arrays are expected to be especially applicable to bright and dynamic events such as micro-explosions and sparks [16].

The creation of twice-ionised species of gases, dications, by electrically or optically triggered breakdown can open new energy harnessing routes as predicted for the $\mathrm{He}_{2}^{2+}$ reaction with hydrogens $\left(\mathrm{H}_{2}, \mathrm{H}_{2}^{+}, \mathrm{H}\right)$, which can release from $18 \mathrm{eV}$ to $43 \mathrm{eV}$ of energy after being supplied with extra energy to surpass the barrier [35]. For dication in the lowest vibrational state, a large kinetic energy is released in a Coulomb explosion-type reaction: $\mathrm{He}_{2}^{2+}+1.28 \mathrm{eV}=2 \mathrm{He}^{+}+10.15 \mathrm{eV}$. This is more than 10 times larger than the energy released by the oxidation of hydrogen: $\mathrm{H}_{2}+\frac{1}{2} \mathrm{O}_{2}=\mathrm{H}_{2} \mathrm{O}+2.52 \mathrm{eV}$. High-intensity ultra-short laser pulses are one of the most established methods to produce multiply charged ions [36]. Since the molecular ions are compact, as is evident from the radius of the $n$th Bohr's orbital $r_{n}=\frac{\hbar}{m_{e} c \alpha} \times \frac{n^{2}}{Z}$, here, $Z$ is the atomic number, $\alpha$ is the fine structure constant, $c$ is speed of light, and $\hbar$ is the small Plank's constant, and $m_{e}$ is the electron mass. Molecular ions can enter solid state hosts as is demonstrated in hydrogen uptake in palladium $\left(\mathrm{H}_{2}^{+}\right.$in $\left.\mathrm{Pd}\right)$ [37]. A solid state matrix could be required for reactions with dications.

Author Contributions: Conceptualisation, S.J., S.H.N. and D.C.; methodology, S.L, S.H.N., J.A., M.B. and J.S.; validation, S.L., S.H.N., D.C., J.D.; investigation, S.L., S.H.N. and S.J.; visualisation, S.L., S.J. and S.H.N.; writing-original draft preparation, S.L., S.J. and S.H.N.; writing-review and editing, all the authors. All authors have read and agreed to the published version of the manuscript.

Funding: This research was funded by the CSIRO Science Industry Endowment Fund (SIEF) STEM+ Researcher in Business and ARC Linkage Project LP190100505.

Institutional Review Board Statement: Not applicable. 
Informed Consent Statement: Not applicable.

Data Availability Statement: The data are not publicly available due to commercial sensitivity.

Acknowledgments: We acknowledge support via strategic operational funding of the Nanotechnology facility at Swinburne University. This work was performed in part at the Melbourne Centre for Nanofabrication (MCN) in the Victorian Node of the Australian National Fabrication Facility (ANFF).

Conflicts of Interest: The authors declare no conflict of interest.

\section{Appendix A. The Black-Body Radiation Temperature}

Figure A1 shows analysis of the $\mathrm{Si} \mu$-thermite without any chemical loading. Time integration of micro-explosion was followed in time with averaging spectra over $10 \mu \mathrm{s}$ windows. The spectra analysed had the highest intensity, which was usually coincident in time with the peak current flow. The best fit of the emission spectra to the black body emission is achieved for the $T=5220 \mathrm{~K}$. The linearised fit provides an estimate of the temperature from the micro-explosion site. Light emission from the expanding plasma region with different density will have spectral intensity profiles affected by re-absorption. The estimate shown in Figure A1 can only serve as a qualitative measure and is based on the short-wavelength part (high photon energy $h v$ ) of emission which can pass through higher density plasma regions. The fit is made through the baseline regions of the characteristic atom/ion peaks. Such a fit is more representative of the black-body emission. Consequently, according to the Wien's displacement law $\lambda_{\text {peak }}=b / T$, where the Wien's displacement constant $b=2898 \mu \mathrm{m} \cdot \mathrm{K}$ the wavelength of the emission maximum is at $\lambda_{\text {peak }}=555 \mathrm{~nm}$ at $T=5220 \mathrm{~K}$. With loaded energetic chemicals, just a slightly higher temperatures were obtained since the maximum of emission was at a shorter wavelength $\lambda_{\text {peak }} \approx 500 \mathrm{~nm}$ (Figure 3a).
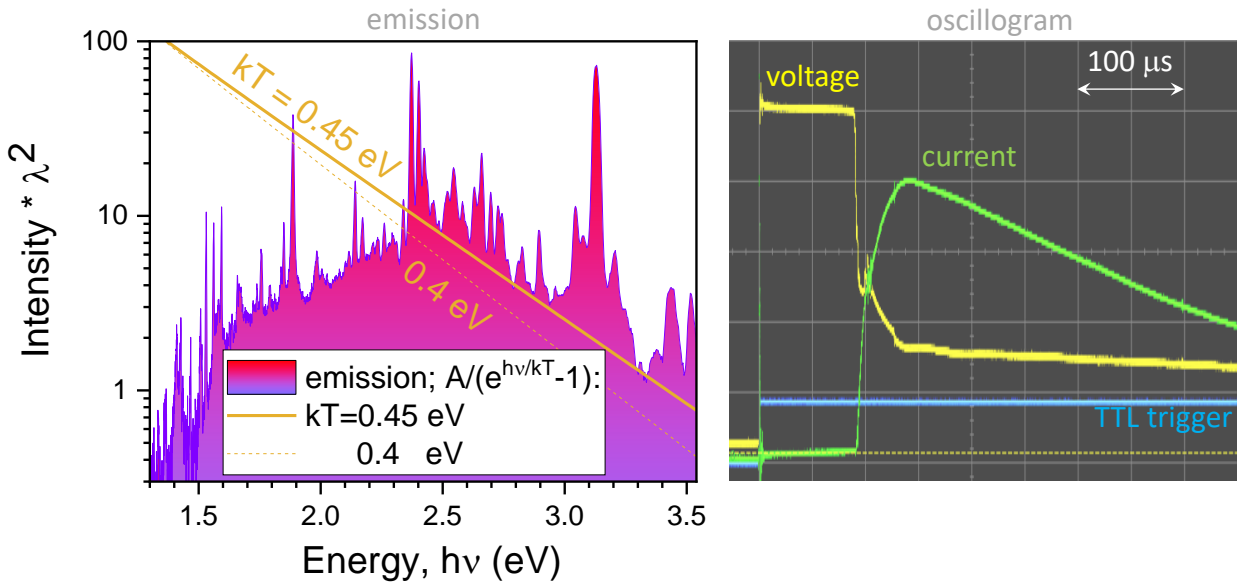

Figure A1. Si $\mu$-thermite (bridge). Estimation of the black body radiation temperature, $T$, from the linearised log-lin emission intensity, $I$, plot $I \lambda^{2} \propto h v$, where $v$ is the photon energy $v=c / \lambda$. The black-body radiation is modeled with $A /\left[\exp \left(\frac{h v}{k T}\right)-1\right]$ function, here $A$ is a fitting amplitude which adjust the vertical shift of the fit-line. The best fit is achieved at $k T=0.45 \mathrm{eV}$ or $T=5220 \mathrm{~K}$ (the dashed line is for the $580 \mathrm{~K}$ lower temperature). Right panel shows the oscilloscope transients for the voltage $U=250 \mathrm{~V}$ and current.

\section{Appendix B. Energy Balance}

Example of TNT $\left(\mathrm{C}_{7} \mathrm{H}_{5} \mathrm{~N}_{3} \mathrm{O}_{6}\right)$ is analysed here. The energy released in a chemical reaction is the heat of explosion $\Delta E=\Delta E_{f}$ (reactant) $-\Delta E_{f}$ (products), where $\Delta E_{f}$ is the heat of formation, which is $-54.39 \mathrm{~kJ} / \mathrm{mol}$ for TNT with a molecular weight of $M=227 \mathrm{~g} / \mathrm{mol}$. For an exothermic (energy release) reaction $\Delta E>0$. Products of reactant are calculated 
following the priority rule: (1) $\mathrm{M}+\mathrm{O}$ metal oxides, (2) $\mathrm{C}+\mathrm{O}$, (3) $2 \mathrm{H}+\mathrm{O}$ (gas), (4) $\mathrm{CO}+\mathrm{O}$, (5) excess $\mathrm{O}, \mathrm{N}, \mathrm{H}$ is counted as fraction of $\mathrm{O}_{2}, \mathrm{~N}_{2}, \mathrm{H}_{2}$ gases. This shows that depositing metals onto $\mu$-thermites is a promising strategy for formation of energetic materials (the priority rules starts with $\mathrm{M}+\mathrm{O}$ reactions), however, oxidiser should be provided. The very same argument holds for porous $\mathrm{Si}$, which is widely used as energetic material. Magnetron sputtering or evaporation by physical vapor deposition (PVD) at different oxygen pressures, is one way to control amount of oxygen in metallic coatings as shown for $\mathrm{Cu}$ (Figure $1 \mathrm{~b})$. The oxygen deficiency is defined later in this section.

The products from TNT explosion then have $6 \mathrm{CO}\left(\mathrm{C}+\mathrm{O}\right.$; step-2 rule), $3 / 2 \mathrm{~N}_{2}$ (from $3 \mathrm{~N}$; step- 5 rule), and similarly $5 / 2 \mathrm{H}_{2}$ (from $5 \mathrm{H}$ ) while one $\mathrm{C}$ is not consumed. The $\mathrm{CO}, \mathrm{CO}_{2}$ and $\mathrm{H}_{2} \mathrm{O}$ are assumed to be in the gaseous form while $\Delta E_{f}$ for $\mathrm{N}_{2}, \mathrm{H}_{2}, \mathrm{O}_{2}$ and all other elements are all zero. Then the reactant-to-product list for the TNT is $\mathrm{C}_{7} \mathrm{H}_{5} \mathrm{~N}_{3} \mathrm{O}_{6} \rightarrow$ $6 \mathrm{CO}(-111.8)+5 / 2(0)+3 / 2(0)+1(0)=-670.8 \mathrm{~kJ} / \mathrm{mol}$, where the heat (enthalpy) of formation of $\mathrm{CO}$ is $\Delta E_{f}=-111.8 \mathrm{~kJ} / \mathrm{mol}$. Finally, the heat of reaction for TNT is $\Delta E=-54.4+670.8=616.4 \mathrm{~kJ} / \mathrm{mol}$ or $2715 \mathrm{~J} / \mathrm{g}$ of energy. It is equivalent to energy $m g h$ for $m=55 \mathrm{~kg}$ drooped from $h=5 \mathrm{~m}$ height; $g=9.8 \mathrm{~m} / \mathrm{s}^{2}$ is the gravity constant. This explains why characterisation of small amount of energetic materials is of paramount importance form safety of testing prospective.

Efficiency of energy release is mainly controlled by the amount of oxygen available for main oxidation reactions. When there is no sufficient oxygen to react with the available $\mathrm{C}$ and $\mathrm{H}$, the explosive is considered to be oxygen deficient and vice versa. The quantitative measure of this is called the oxygen balance $O B=-M_{W}(O) / M_{W}$ (reactant $) \times\left(2 \mathrm{C}+\frac{1}{2} \mathrm{H}+\right.$ $\mathrm{M}-\mathrm{O}$ ) $\%$, where the molecular weight of oxygen $M_{W}(\mathrm{O})=16 \mathrm{~g} / \mathrm{mol}, \mathrm{C}, \mathrm{H}, \mathrm{M}, \mathrm{O}$ are the number of moles of carbon, hydrogen, metal and oxygen, respectively. For the TNT, $\mathrm{OB}=-(16 / 227)[2(7)+5 / 2-6]=-72 \%$ shows that it is oxygen deficient. The optimal performance with maximum energy release is when $O B=0 \%$.

The conversion of the heat of explosion $\Delta E$ into a mechanical work is determined by the amount of product gases available for expansion. In the case of TNT, 10 moles of gas are produced for each mole of explosive. The Berthelot approximation is used to compare different chemicals. It states that the relative explosive strength $R S$ of an explosive (compared to TNT) is calculated as $R S=840 \Delta n \Delta E / M_{W}^{2} \%$, where $\Delta n$ is the number of moles of gas per mole of explosive and the factor of 840 accounts for the units and values of $\Delta E$ and $\Delta n$ for TNT.

The calculated $R S$ is of a limited use and there are number of standard tests to directly measure the work performed. The presented analysis and definitions can be used to compare reactivity of different materials, production of gaseous products and to estimate oxygen deficiency in a smaller and scalable method.

\section{References}

1. Betti, R.; Hurricane, O.A. Inertial-confinement fusion with lasers. Nat. Phys. 2016, 12, 435-448. [CrossRef]

2. Mourou, G.; Tajima, T.; Bulanov, S. Optics in the relativistic regime. Rev. Mod. Phys. 2006, 78, 309-371. [CrossRef]

3. Gibbon, P.; Förster, E. Short-pulse laser-plasma interactions. Plasma Phys. Control. Fusion 1996, 38, 769-793. [CrossRef]

4. Torretti, F.; Sheil, J.; Schupp, R.; Basko, M.M.; Bayraktar, M.; Meijer, R.A.; Witte, S.; Ubachs, W.; Hoekstra, R.; Versolato, O.O.; et al. Prominent radiative contributions from multiply-excited states in laser-produced tin plasma for nanolithography. Nat. Commun. 2020, 11, 2334. [CrossRef] [PubMed]

5. Gorbunov, L.M.; Kirsanov, V.I. Excitation of plasma waves by an electromagnetic wave packet. Sov. Phys. JETP 1987, 66, 290-294.

6. Schwoerer, H. Particle acceleration with lasers. S. Afr. J. Sci. 2008, 104, 299-304.

7. Charles, C. Plasmas for spacecraft propulsion. J. Phys. D Appl. Phys. 2009, 42, 163001. [CrossRef]

8. Gamaly, E.G.; Rode, A.V. Ultrafast re-structuring of the electronic landscape of transparent dielectrics: New material states (Die-Met). Appl. Phys. A 2018, 124, 278. [CrossRef]

9. Luo, Z.; Li, D.; Su, B.; Zhang, S.; Deng, J. On the time coupling analysis of explosion pressure and intermediate generation for multiple flammable gases. Energy 2020, 198, 117329. [CrossRef]

10. Maurice, S.; Clegg, S.M.; Wiens, R.C.; Gasnault, O.; Rapin, W.; Forni, O.; Cousin, A.; Sautter, V.; Mangold, N.; Le Deit, L.; et al. ChemCam activities and discoveries during the nominal mission of the Mars Science Laboratory in Gale crater, Mars. J. Anal. At. Spectrom. 2016, 31, 863-889. [CrossRef]

11. Lasue, J.; Wiens, R.C.; Clegg, S.M.; Vaniman, D.T.; Joy, K.H.; Humphries, S.; Mezzacappa, A.; Melikechi, N.; McInroy, R.E.; Bender, S. Remote laser-induced breakdown spectroscopy (LIBS) for lunar exploration. J. Geophys. Res. 2012, 117, E01002. [CrossRef]

12. Russo, R.; Mao, X.; Liu, H.; Yoo, J.; Mao, S. Time-resolved plasma diagnostics and mass removal during single-pulse laser ablation. Appl. Phys. A 1999, 69, S887-S894. [CrossRef] 
13. Baginski, T.A.; Systems, R.; Taliaferro, S.L.; Fahey, W.D. Novel Electroexplosive Device Incorporating a Reactive Laminated Metallic Bridge. J. Propuls. Power 2001, 17, 184-189. [CrossRef]

14. Marín, L.; Nanayakkara, C.E.; Veyan, J.F.; Warot-Fonrose, B.; Joulie, S.; Esteìve, A.; Tenailleau, C.; Chabal, Y.J.; Rossi, C. Enhancing the Reactivity of $\mathrm{Al} / \mathrm{CuO}$ Nanolaminates by $\mathrm{Cu}$ Incorporation at the Interfaces. ACS Appl. Mater. Interfaces 2015. [CrossRef]

15. Lundgaard, S.; Ng, S.H.; Cahill, D.; Dahlberg, J.; Ruan, D.; Cole, N.; Stoddart, P.R.; Juodkazis, S. Towards Safer Primers: A Review. Technologies 2019, 7, 75. [CrossRef]

16. Anand, V.; Ng, S.H.; Katkus, T.; Juodkazis, S. Spatio-Spectral-Temporal Imaging of Fast Transient Phenomena Using a Random Array of Pinholes. Adv. Photonics Res. 2020, 1, 2000032.

17. Atomic Spectra Database. NIST Standard Reference Database 78 (Ver. 5.8). Nat. Inst. Stand. Technol. 2020.. [CrossRef]

18. Effenberger, A.J., Jr.; Scott, J.R. Effect of Atmospheric Conditions on LIBS Spectra. Sensors 2010, 10, 4907-4925. [CrossRef]

19. Kaladè, J.; Rinkunas, R.; Purlys, R.; Žilinskas, P.J.; Lozovski, T.; Poškus, A. The Dynamics of the Electric Field Distribution in the Surface of Insulating Film Irradiated by Air Ions. Mater. Sci. 2016, 22, 126-131. [CrossRef]

20. Levchenko, I.; Xu, S.; Cherkun, O.; Baranov, O.; Bazaka, K. Plasma meets metamatertials: Three ways to advance space micropropulsion systems. Adv. Phys. X 2020, 6, 1834452.

21. Juodkazis, S.; Murazawa, N.; Wakatsuki, H.; Misawa, H. Laser irradiation induced disintegration of a bubble in a glass melt. Appl. Phys. A 2007, 87, $41-45$. [CrossRef]

22. Laux, C.; Spence, T.; Kruger, C.; Zare, R. Optical diagnostics of atmospheric pressure air plasmas. Plasma Sources Sci. Technol. 2003, 12, 125-138. [CrossRef]

23. Pawłat, J.; Terebun, P.; Kwiatkowski, M.; Tarabová, B.; Koval'ová, Z.; Kučerová, K.; Machala, Z.; Janda, M.; Hensel, K. Evaluation of Oxidative Species in Gaseous and Liquid Phase Generated by Mini-Gliding Arc Discharge. Plasma Chem. Plasma Process. 2019, 39, 627-642. [CrossRef]

24. Lee, J.; Kim, T.; Ryu, S.U.; Choi, K.; Ahn, G.H.; Paik, J.G.; Ryu, B.; Park, T.; Won, Y.S. Study on the Aging Mechanism of Boron Potassium Nitrate (BKNO3) for Sustainable Efficiency in Pyrotechnic Mechanical Devices. Sci. Rep. 2018, 8, 1-9. [CrossRef]

25. Žukauskas, A.; Malinauskas, M.; Kadys, A.; Gervinskas, G.; Seniutinas, G.; Kandasamy, S.; Juodkazis, S. Black silicon: Substrate for laser 3D micro/nano-polymerization. Opt. Express 2013, 21, 6901-6909. [CrossRef]

26. Gervinskas, G.; Seniutinas, G.; Hartley, J.S.; Kandasamy, S.; Stoddart, P.R.; Juodkazis, S. Surface-enhanced Raman scattering sensing on black silicon. Annal. Phys. 2013, 525, 907-914. [CrossRef]

27. Federation of American Scientists. Military Analysis Network. ES310. Introduction to Naval Weapons Engineering. Course Syllabus: Chemistry. 1998. Available online: https://fas.org/man/dod-101/navy/docs/es310/chemstry/chemstry.htm (accessed on 1 January 2021).

28. Sinha, S.; Piekiel, N.; Smith, G.; Morris, C. Investigating aging effects for porous silicon energetic materials. Combust. Flame 2017, 181, 164-171. [CrossRef]

29. Hora, H. Laser Plasma Physics: Forces and the Nonlinearity Principle; SPIE Press: Bellingham, WA, USA, 2000.

30. Fujisawa, H.; Ryu, M.; Lundgaard, S.; Linklater, D.; Ivanova, E.; Nishijima, Y.; Juodkazis, S.; Morikawa, J. Direct Measurement of Temperature Diffusivity of Nanocellulose-Doped Biodegradable Composite Films. Micromachines 2011, 11, 738. [CrossRef]

31. Cebe, P.; Hu, X.; Kaplan, D.; Zhuravlev, E.; Wurm, A.; Arbeiter, D.; Schick, C. Beating the Heat—Fast Scanning Melts Silk Beta Sheet Crystals. Sci. Rep. 2013, 3, 1130. [CrossRef]

32. Minakova, A.; Morikawa, J.; Ryu, M.; Zhuravlev, E.; Schick, C. Variations of interfacial thermal conductance at melting and crystallization of an indium micro-particle in contact with a solid. Mater. Des. 2021, 201, 109475. [CrossRef]

33. Lundgaard, S.; Ng, S.H.; Nishijima, Y.; Mazilu, M.; Juodkazis, S. Black Metals: Optical Absorbers. Micromachines 2020, 11, 256. [CrossRef] [PubMed]

34. Toulouse, A.; Drozella, J.; Thiele, S.; Giessen, H.; Herkommer, A. 3D-printed miniature spectrometer for the visible range with a $100 \times 100 \mu \mathrm{m}^{2}$ footprint. Light Adv. Manuf. 2020,1, 5 .

35. Falcinelli, S.; Rosi, M. Production and Characterization of Molecular Dications: Experimental and Theoretical Efforts. Molecules 2020, 25, 4157. [CrossRef] [PubMed]

36. Mathur, D. Structure and dynamics of molecules in high charge states. Phys. Rep. 2004, 391, 1-1187. [CrossRef]

37. Juodkazis, K.; Juodkazyte, J.; Grigucevičienè, A.; Juodkazis, S. Hydrogen species within the metals: Role of molecular hydrogen ion $\mathrm{H}_{2}^{+}$. Appl. Surf. Sci. 2011, 258, 743-747. [CrossRef] 